\title{
Research Article \\ Stabilization and Observability of a Rotating Timoshenko Beam Model
}

\author{
Alexander Zuyev and Oliver Sawodny \\ Received 30 September 2006; Revised 12 February 2007; Accepted 13 May 2007 \\ Recommended by José Manoel Balthazar
}

A control system describing the dynamics of a rotating Timoshenko beam is considered. We assume that the beam is driven by a control torque at one of its ends, and the other end carries a rigid body as a load. The model considered takes into account the longitudinal, vertical, and shear motions of the beam. For this distributed parameter system, we construct a family of Galerkin approximations based on solutions of the homogeneous Timoshenko beam equation. We derive sufficient conditions for stabilizability of such finite dimensional system. In addition, the equilibrium of the Galerkin approximation considered is proved to be stabilizable by an observer-based feedback law, and an explicit control design is proposed.

Copyright () 2007 A. Zuyev and O. Sawodny. This is an open access article distributed under the Creative Commons Attribution License, which permits unrestricted use, distribution, and reproduction in any medium, provided the original work is properly cited.

\section{Introduction}

Control issues for several models of flexible manipulators have been intensively studied by many authors. A particular list of references in this area can be found in monographs $[1,2]$. There are two common approaches to represent the motion of such manipulators. The first approach deals with systems of rigid bodies [3], Galerkin approximations [4, 5], or finite element methods [6] to derive mathematical models with finite degrees of freedom. The second approach treats a manipulator as a distributed parameter system. The majority of publications in this distributed parameter approach are concentrated on the Euler-Bernoulli beam model (see [7], [8, Chapter 10.8], [1, Chapter 4], [2, 9, 10]).

A possible extension of the Euler-Bernoulli model was proposed by Timoshenko [11]. From the engineering viewpoint, the Timoshenko beam has an advantage of describing the effects of rotary inertia and the deflection due to shear. Control of Timoshenko beams was studied in [12-17], [1, Chapter 5.1.2]. The motion of a payload, usually attached to a 
real manipulator, is neglected in all these publications. In [18], a clamped beam with an end mass is proved to be stabilizable by a feedback control applied to the tip. The author of [19] addresses the development of LQR techniques and computation algorithms for beams with controlling torques applied to the hub. A limitation of these results is that a knowledge of the full infinite dimensional space is required. In [5], a hybrid system of partial and ordinary differential equations, representing the oscillations of a flexible beam, has been studied for the case when the control is the acceleration at a point. We have considered a model for the vertical motion of a beam and estimated its physical parameters from measurements of modal frequencies in [20].

It should be emphasized that, in contrast to the above publications, we study here a rotating beam that carries a payload under the action of gravity, the control torque is applied at the hub, and the longitudinal motion is taken into account. The motivation for this study is to control the motion of a real flexible-link manipulator-turntable ladder. Such a turntable ladder has been described in [3], where a dynamical model with two rigid bodies (two degrees of freedom) has been used to represent the first mode of oscillations.

This paper is organized as follows. In Section 2, we derive the motion equations for a flexible beam with a load under the action of gravity and the control torque. Section 3 contains necessary details for computing the eigenvalues and eigenfunctions of the associated Sturm-Liouville problem. By using Galerkin's method, we approximate the dynamics by a system of ODEs in Section 4. In the derivation procedure, we exploit the variational form instead of taking the standard inner product in $L^{2}$. The order of approximation may be chosen arbitrarily. In Section 5, a state feedback control which stabilizes the equilibrium of the Galerkin approximation is obtained (Theorem 5.1). In order to justify a possibility of implementation of the controller proposed, we study the observability problem in Section 6. The closed-loop system is proved to be asymptotically stable, provided that the feedback is generated by a Luenberger-type observer (Theorem 6.2). The proof of Theorem 6.2 is based on the invariance principle. The main advantage of our approach is that the control design is done explicitly; all the parameters appearing in the feedback law and dynamical observer can be effectively computed through integral moments with respect to solutions of the Sturm-Liouville problem. It is also important that no derivatives of the input signals are needed for the state estimation.

\section{Description of the model}

Consider a flexible beam rotating in the vertical plane around the fixed point $O$ (see Figure 2.1).

We assume that the beam is driven by a control torque $M$ at one of its ends (the hub at $O$ ), and the other end (point $C$ ) carries a payload of mass $m$.

Let $l$ be the length of the beam. We assume that the centerline of the beam in its undeformed reference configuration occupies the segment $[0, l]$ on the $O x$-axis. Consider a particle $P$ on the centerline and denote by $x$ its coordinate in the reference configuration. At a given time $t$, let $(x+s(x, t), w(x, t))$ be the coordinates of the position vector for $P$ in the Cartesian frame $O x y$. We introduce the notation $\psi(x, t)$ for the rotation angle of the cross section area at $P$ due to bending. By taking into account the longitudinal, vertical, 


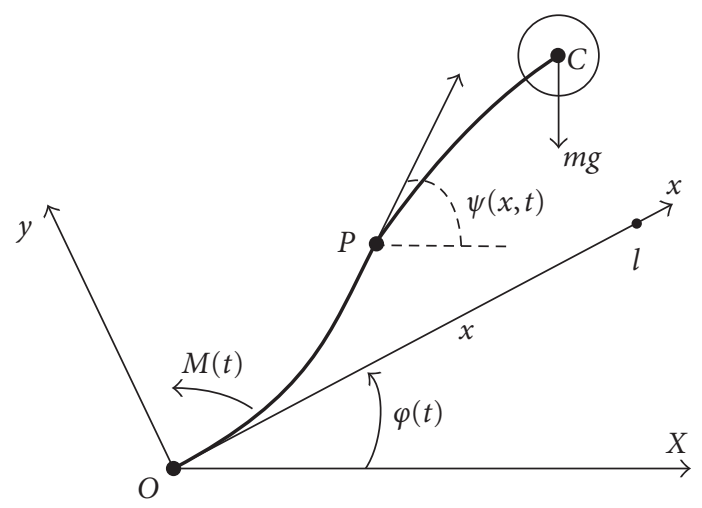

Figure 2.1. A rotating Timoshenko beam.

and shear motions, we derive the following expression for the kinetic energy of the system considered:

$$
\begin{aligned}
2 T= & \int_{0}^{l}\left\{\rho(x)\left[(\dot{w}+x \dot{\varphi})^{2}+(w \dot{\varphi})^{2}+\dot{s}^{2}+2 \dot{\varphi}(\dot{w} s-\dot{s} w)+(\dot{\varphi})^{2}(s+2 x) s\right]+I_{\rho}(x)(\dot{\varphi}+\dot{\psi})^{2}\right\} d x \\
& +J_{0}(\dot{\varphi})^{2}+\left.m\left\{(\dot{w}+x \dot{\varphi})^{2}+(w \dot{\varphi})^{2}+\dot{s}^{2}+2 \dot{\varphi}(\dot{w} s-\dot{s} w)+(\dot{\varphi})^{2}(s+2 x) s\right\}\right|_{x=l} \\
& +\left.J_{c}\{\dot{\varphi}+\dot{\psi}\}^{2}\right|_{x=l},
\end{aligned}
$$

where $\varphi(t)$ is the angle between the moving axis $O x$ and the horizontal direction, $\rho(x)$ is the mass per unit length of the beam, $I_{\rho}(x)$ is the mass moment of inertia of the cross section, and $J_{0}$ is the hub moment of inertia. The mass distribution for the payload is characterized by the moment of inertia $J_{c}$ with respect to its center of mass $C$.

In this paper, we use dots to denote derivatives with respect to time $t$, and primes to denote derivatives with respect to the space variable $x$.

Assuming that the beam is inextensible, we get the following relation on $w$ and $s$ :

$$
s^{\prime}=-\frac{1}{2} w^{\prime 2}+o\left(w^{\prime 2}\right)
$$

The integration of this relation, with the higher order terms being omitted, yields

$$
s(x, t)=-\frac{1}{2} \int_{0}^{x} w^{\prime 2}(\xi, t) d \xi
$$

We assume that the deformation of the beam is small and drop the terms of order higher than 2 relative to $w$ when computing the Lagrangian of the system considered. 
Following the Timoshenko beam model [11], [7, page 1142], and exploiting (2.1), (2.3), the Lagrangian takes the form

$$
\begin{aligned}
2 L= & \int_{0}^{l}\left\{\rho(x)\left((\dot{w}+x \dot{\varphi})^{2}+\dot{\varphi}^{2} w^{2}\right)-\rho_{2}(x) \dot{\varphi}^{2} w^{\prime 2}+I_{\rho}(\dot{\varphi}+\dot{\psi})^{2}-K\left(\psi-w^{\prime}\right)^{2}-E I\left(\psi^{\prime}\right)^{2}\right\} d x \\
& +m\left\{\dot{\varphi}^{2} w^{2}(l, t)+(l \dot{\varphi}+\dot{w}(l, t))^{2}\right\}+J_{c}\{\dot{\varphi}+\dot{\psi}(l, t)\}^{2}+J_{0} \dot{\varphi}^{2} \\
& -g \int_{0}^{l}\left\{\left(2 \rho x-\rho_{1} w^{\prime 2}\right) \sin \varphi+2 \rho w \cos \varphi\right\} d x-2 m g\{l \sin \varphi+w(l, t) \cos \varphi\},
\end{aligned}
$$

where

$$
\rho_{1}(x)=\int_{x}^{l} \rho(\xi) d \xi+m, \quad \rho_{2}(x)=\int_{x}^{l} \xi \rho(\xi) d \xi+m l .
$$

Here $E$ and $I$ are Young's modulus and the moment of inertia of the cross section of the beam, respectively, $g$ is the acceleration of gravity. The coefficient $K$ is equal to $k G A$, where $G$ is the modulus of elasticity in shear, $A$ is the cross sectional area, and $k$ is a constant depending on the shape of the cross section. We assume that $\rho, I_{\rho}, E I$, and $K$ are all positive, differentiable functions of the space variable $x$.

If $C^{2}$-functions $(\varphi(t), w(x, t), \psi(x, t))$ define the motion of the system for the control torque $M(t)$ on a segment $t \in\left[t_{1}, t_{2}\right]$ then Hamilton's principle yields

$$
\delta\left(\int_{t_{1}}^{t_{2}} L d t\right)+\int_{t_{1}}^{t_{2}} M(t) \delta \varphi(t) d t=0
$$

for any admissible variations $(\delta \varphi(t), \delta w(x, t), \delta \psi(x, t))$ satisfying the boundary conditions

$$
\begin{gathered}
\left.\delta \varphi\right|_{t=t_{1}}=\left.\delta \varphi\right|_{t=t_{2}}=0,\left.\quad \delta w\right|_{t=t_{1}}=\left.\delta w\right|_{t=t_{2}}=0,\left.\quad \delta \psi\right|_{t=t_{1}}=\left.\delta \psi\right|_{t=t_{2}}=0, \\
\left.\delta w\right|_{x=0}=0,\left.\quad \delta \psi\right|_{x=0}=0 .
\end{gathered}
$$

By computing the first variation in (2.6) and integrating by parts, we get

$$
\int_{t_{1}}^{t_{2}}\left\{\left(M+\frac{\partial L}{\partial \varphi}-\frac{d}{d t} \frac{\partial L}{\partial \dot{\varphi}}\right) \delta \varphi(t)-\mu(\delta w(\cdot, t), \delta \psi(\cdot, t) ; \varphi, w, \psi)\right\} d t=0,
$$

where the functional $\mu$ is linear with respect to $\delta w$ and $\delta \psi$ :

$$
\begin{aligned}
& \mu(\delta w(\cdot, t), \delta \psi(\cdot, t) ; \varphi, w, \psi) \\
& =\int_{0}^{l} \delta w(x, t)\left\{\left(\ddot{w}+x \ddot{\varphi}-(\dot{\varphi})^{2} w+g \cos \varphi\right) \rho+\left(K\left(\psi-w^{\prime}\right)+\left(g \rho_{1} \sin \varphi-(\dot{\varphi})^{2} \rho_{2}\right) w^{\prime}\right)^{\prime}\right\} d x \\
& \quad+\int_{0}^{l} \delta \psi(x, t)\left\{I_{\rho}(\ddot{\psi}+\ddot{\varphi})+K\left(\psi-w^{\prime}\right)-\left(E I \psi^{\prime}\right)^{\prime}\right\} d x+\left.\delta \psi(l, t)\left\{J_{c}(\ddot{\varphi}+\ddot{\psi})+E I \psi^{\prime}\right\}\right|_{x=l} \\
& \quad+\left.\delta w(l, t)\left\{K\left(w^{\prime}-\psi\right)+m\left(\ddot{w}+l \ddot{\varphi}-(\dot{\varphi})^{2} w+g \cos \varphi\right)+m\left(l(\dot{\varphi})^{2}-g \sin \varphi\right) w^{\prime}\right\}\right|_{x=l} .
\end{aligned}
$$


Thus, as (2.8) vanishes on each admissible variation satisfying (2.7), we get the following boundary value problem:

$$
\begin{aligned}
& \ddot{w} \frac{1}{\rho}\left(K\left(\psi-w^{\prime}\right)\right)^{\prime}=-g \cos \varphi-x \ddot{\varphi}+\dot{\varphi}^{2} w+\frac{1}{\rho}\left(\left(\rho_{2} \dot{\varphi}^{2}-g \rho_{1} \sin \varphi\right) w^{\prime}\right)^{\prime} ; \\
& \ddot{\psi}+ \frac{K}{I_{\rho}}\left(\psi-w^{\prime}\right)-\frac{1}{I_{\rho}}\left(E I \psi^{\prime}\right)^{\prime}=-\ddot{\varphi}, \quad x \in(0, l) ; \\
&\left.w\right|_{x=0}=\left.\psi\right|_{x=0}=0 ; \\
&\left.K\left(\psi-w^{\prime}\right)\right|_{x=l}=\left.m\left\{\ddot{w}+l \ddot{\varphi}-\dot{\varphi}^{2} w+g \cos \varphi+\left(l \dot{\varphi}^{2}-g \sin \varphi\right) w^{\prime}\right\}\right|_{x=l} ; \\
&-\left.E I \psi^{\prime}\right|_{x=l}=J_{c}\left(\ddot{\varphi}+\left.\ddot{\psi}\right|_{x=l}\right), \\
& M(t)=\frac{d}{d t} \frac{\partial L}{\partial \dot{\varphi}}-\frac{\partial L}{\partial \varphi}=\left\{J_{c}+J_{0}+m\left[l^{2}+w^{2}(l, t)\right]+\int_{0}^{l}\left[I_{\rho}+\left(x^{2}+w^{2}\right) \rho-\rho_{2} w^{\prime 2}\right] d x\right\} \ddot{\varphi} \\
& \quad+\int_{0}^{l}\left(\rho x \ddot{w}+I_{\rho} \ddot{\psi}+2 \rho w \dot{\varphi} \dot{w}-2 \rho_{2} w^{\prime} \dot{\varphi} \dot{w}^{\prime}\right) d x+\left.m(l \ddot{w}+2 w \dot{\varphi} \dot{w})\right|_{x=l}+\left.J_{c} \ddot{\psi}\right|_{x=l} \\
& \quad+g\left\{\int_{0}^{l}\left(\rho x-\frac{1}{2} \rho_{1} w^{\prime 2}\right) d x+m l\right\} \cos \varphi-g\left\{\int_{0}^{l} \rho w d x+m w(l, t)\right\} \sin \varphi .
\end{aligned}
$$

Straightforward computations show that the above control system admits an equilibrium

$$
\varphi(t)=\varphi_{0}, \quad w(x, t)=w_{0}(x), \quad \psi(x, t)=\psi_{0}(x), \quad M(t)=M_{0}
$$

if and only if the following conditions are satisfied:

$$
\begin{aligned}
& \left(K\left(w_{0}^{\prime}(x)-\psi_{0}(x)\right)\right)^{\prime}=g\left\{\rho \cos \varphi_{0}+\left(\rho_{1} w_{0}{ }^{\prime}\right)^{\prime} \sin \varphi_{0}\right\} \\
& \left(E I \psi_{0}^{\prime}(x)\right)^{\prime}+K\left(w_{0}^{\prime}(x)-\psi_{0}(x)\right)=0, \quad x \in(0, l) ; \\
& w_{0}(0)=\psi_{0}(0)=0 ; \quad \psi_{0}^{\prime}(l)=0 ; \quad K\left(\psi_{0}(l)-w_{0}^{\prime}(l)\right)=m g\left(\cos \varphi_{0}-w_{0}^{\prime}(l) \sin \varphi_{0}\right) ; \\
& \frac{M_{0}}{g}=\left(\int_{0}^{l}\left(\rho x-\frac{1}{2} \rho_{1} w_{0}^{\prime 2}\right) d x+m l\right) \cos \varphi_{0}-\left(\int_{0}^{l} \rho w_{0} d x+m w_{0}(l)\right) \sin \varphi_{0} .
\end{aligned}
$$

Our goal is to control the system (2.10) around its steady state (2.12).

\section{Perturbed dynamics}

Let $\left(\varphi_{0}, w_{0}, \psi_{0}\right)$ be a solution of $(2.12)$ with some $M_{0}$. Then plugging

$$
\varphi=\varphi_{0}+\tilde{\varphi}, \quad w=w_{0}+\widetilde{w}, \quad \psi=\psi_{0}+\widetilde{\psi}, \quad M=M_{0}+\widetilde{M}
$$


into the dynamical equations (2.10) yields the following control system:

$$
\begin{gathered}
\ddot{\tilde{\varphi}}=v ; \\
\ddot{\widetilde{w}}+\frac{1}{\rho}\left(K\left(\widetilde{\psi}-\widetilde{w}^{\prime}\right)\right)^{\prime}=-x v+g \tilde{\varphi} \sin \varphi_{0}-\frac{g}{\rho}\left(\left(\tilde{w}^{\prime} \sin \varphi_{0}+\tilde{\varphi} w_{0}^{\prime} \cos \varphi_{0}\right) \rho_{1}\right)^{\prime}+\cdots ; \\
I_{\rho} \ddot{\widetilde{\psi}}+K\left(\tilde{\psi}-\tilde{w}^{\prime}\right)-\left(E I \tilde{\psi}^{\prime}\right)^{\prime}=-I_{\rho} v ; \\
\left.\tilde{w}\right|_{x=0}=\left.\tilde{\psi}\right|_{x=0}=0 ; \\
\left.\left(\frac{K}{m}\left(\widetilde{w}^{\prime}-\tilde{\psi}\right)+\ddot{\widetilde{w}}-g\left(\sin \varphi_{0}+w_{0}^{\prime} \cos \varphi_{0}\right) \tilde{\varphi}-g \tilde{w}^{\prime} \sin \varphi_{0}+\cdots\right)\right|_{x=l}=-l v ; \\
\left.\left(E I \widetilde{\psi}^{\prime}+J_{c} \ddot{\widetilde{\psi}}\right)\right|_{x=l}=-J_{c} v,
\end{gathered}
$$

where

$$
\begin{aligned}
v=\left(J_{0}\right. & \left.+\int_{0}^{l}\left[\left(w_{0}\right)^{2} \rho-\left(w_{0}^{\prime}\right)^{2} \rho_{2}\right] d x+m w_{0}^{2}(l)\right)^{-1} \\
& \times\left\{\widetilde{M}+g\left(\int_{0}^{l}\left[\left(\rho w_{0}-\rho_{1} w_{0}^{\prime}\right) \cos \varphi_{0}-\frac{1}{2} \rho_{1}\left(w_{0}^{\prime}\right)^{2} \sin \varphi_{0}\right] d x+m w_{0}(l) \cos \varphi_{0}\right) \tilde{\varphi}\right. \\
& \left.+g \int_{0}^{l}\left[\left(w_{0}^{\prime} \cos \varphi_{0}-\sin \varphi_{0}\right) \rho_{1} \widetilde{w}^{\prime}+\rho \tilde{w} \sin \varphi_{0}\right] d x+m g \tilde{w}(l, t)-E I \widetilde{\psi}^{\prime}(0, t)\right\}+\cdots,
\end{aligned}
$$

where the symbol "..." denotes terms of order of smallness 2 or higher with respect to $\tilde{\varphi}, \tilde{w}, \tilde{\psi}$ and their derivatives.

As, for each state $(\widetilde{\varphi}(t), \dot{\widetilde{\varphi}}(t), \widetilde{w}(\cdot, t), \dot{\widetilde{w}}(\cdot, t), \widetilde{\psi}(\cdot, t), \dot{\widetilde{\psi}}(\cdot, t))$, there is a one-to-one correspondence between $\widetilde{M}$ and $v$, we may treat $v$ as a control in (3.2) and assume that it may take any value in $\mathbb{R}$.

3.1. Separation of variables. To derive a finite dimensional approximation, let us first study solutions of the control system (3.2) of a particular form

$$
\tilde{\varphi}(t) \equiv 0, \quad \widetilde{w}(x, t)=\bar{w}(x) q(t), \quad \widetilde{\psi}(x, t)=\bar{\psi}(x) q(t) .
$$

By substituting the above relations into (3.2), we get $\ddot{q}(t)=-\lambda q(t)$ together with the following Sturm-Liouville problem:

$$
\begin{gathered}
\left(K\left(\bar{\psi}-\bar{w}^{\prime}\right)+g \rho_{1} \bar{w}^{\prime} \sin \varphi_{0}\right)^{\prime}-\lambda \rho \bar{w}=0, \\
K\left(\bar{\psi}-\bar{w}^{\prime}\right)-\left(E I \bar{\psi}^{\prime}\right)^{\prime}-\lambda I_{\rho} \bar{\psi}=0, \quad x \in(0, l), \\
\bar{w}(0)=0, \quad \bar{\psi}(0)=0, \\
K\left(\bar{w}^{\prime}(l)-\bar{\psi}(l)\right)-m g \bar{w}^{\prime}(l) \sin \varphi_{0}-m \lambda \bar{w}(l)=0, \\
E I \bar{\psi}^{\prime}(l)-\lambda J_{c} \bar{\psi}(l)=0,
\end{gathered}
$$

where $\lambda$ is a scalar parameter. 
3.2. Eigenvalues of the Sturm-Liouville problem. Let

$$
\mathscr{H}=\left\{\left(\begin{array}{l}
\bar{w} \\
\bar{\psi}
\end{array}\right): \bar{w} \in H^{1}[0, l], \bar{\psi} \in H^{1}[0, l], \bar{w}(0)=\bar{\psi}(0)=0\right\}
$$

where $H^{1}[0, l]$ is the Sobolev space. Consider the following symmetric positive definite bilinear form on $\mathcal{H}$ :

$$
\left\langle\begin{array}{ll}
w_{1} & w_{2} \\
\psi_{1}^{\prime} & \psi_{2}
\end{array}\right\rangle_{\mathscr{H}}=\int_{0}^{l}\left(\rho w_{1} w_{2}+I_{\rho} \psi_{1} \psi_{2}\right) d x+m w_{1}(l) w_{2}(l)+J_{c} \psi_{1}(l) \psi_{2}(l)
$$

A straightforward consequence of the above definition is the following.

Lemma 3.1. Let $\left(\lambda_{1}, w_{1}, \psi_{1}\right)$ and $\left(\lambda_{2}, w_{2}, \psi_{2}\right)$ be nontrivial solutions of (3.5). Then

$$
\left\langle\begin{array}{ll}
w_{1} & w_{2} \\
\psi_{1}, & \psi_{2}
\end{array}\right\rangle_{\mathscr{H}}=0 \quad \text { if } \lambda_{1} \neq \lambda_{2}
$$

Moreover, if $K(x)=$ const and

$$
\frac{2\left(m+\int_{0}^{l} \rho d x\right) g \sin \varphi_{0}}{K} \leq 1, \quad \frac{K l^{2}}{E I} \leq 2
$$

then all eigenvalues $\lambda$ of (3.5) are nonnegative real numbers.

Proof. If $\left(\lambda_{1}, w_{1}, \psi_{1}\right)$ is a solution of (3.5) then

$$
\begin{aligned}
& \lambda_{1}\left\langle\begin{array}{ll}
w_{1} & w_{2} \\
\psi_{1}, & \psi_{2}
\end{array}\right\rangle_{\mathscr{H}}=\left\langle\begin{array}{ll}
\lambda_{1} w_{1} & w_{2} \\
\lambda_{1} \psi_{1} & \psi_{2}
\end{array}\right\rangle_{\mathscr{H}} \\
& \quad=\int_{0}^{l}\left(K\left(\psi_{1}-w_{1}^{\prime}\right)+g \rho_{1} w_{1}^{\prime} \sin \varphi_{0}\right)^{\prime} w_{2} d x+\int_{0}^{l}\left(K\left(\psi_{1}-w_{1}^{\prime}\right)-\left(E I \psi_{1}^{\prime}\right)^{\prime}\right) \psi_{2} d x \\
& +\left(K\left(w_{1}^{\prime}(l)-\psi_{1}(l)\right)-m g w_{1}^{\prime}(l) \sin \varphi_{0}\right) w_{2}(l)+E I \psi_{1}^{\prime}(l) \psi_{2}(l) .
\end{aligned}
$$

Performing integration by parts in the above expression, we get

$$
\lambda_{1}\left\langle\begin{array}{ll}
w_{1} & w_{2} \\
\psi_{1} & \psi_{2}
\end{array}\right\rangle_{\mathscr{H}}=\int_{0}^{l}\left\{K\left(w_{1}^{\prime} w_{2}^{\prime}+\psi_{1} \psi_{2}-w_{1}^{\prime} \psi_{2}-w_{2}^{\prime} \psi_{1}\right)+E I \psi_{1}^{\prime} \psi_{2}^{\prime}-g \rho_{1} w_{1}^{\prime} w_{2}^{\prime} \sin \varphi_{0}\right\} d x
$$


The permutation of arguments in (3.11) yields

$$
\lambda_{2}\left\langle\begin{array}{ll}
w_{1} & w_{2} \\
\psi_{1}^{\prime} & \psi_{2}
\end{array}\right\rangle_{\mathscr{H}}=\lambda_{2}\left\langle\begin{array}{ll}
w_{2} & w_{1} \\
\psi_{2}^{\prime} & \psi_{1}
\end{array}\right\rangle_{\mathscr{H}}=\lambda_{1}\left\langle\begin{array}{ll}
w_{1} & w_{2} \\
\psi_{1}^{\prime} & \psi_{2}
\end{array}\right\rangle_{\mathscr{H}} .
$$

Hence, $\left\langle\begin{array}{ll}w_{1} & w_{2} \\ \psi_{1}, & \psi_{2}\end{array}\right\rangle_{\mathscr{H}}=0$ if $\lambda_{1} \neq \lambda_{2}$. If $w_{2}=w_{1}$ and $\psi_{2}=\psi_{1}$ then $(3.11)$ implies

$$
\begin{aligned}
\lambda_{1}\left\langle\begin{array}{ll}
w_{1} & w_{1} \\
\psi_{1}, & \psi_{1}
\end{array}\right\rangle_{\mathscr{H}} & =\int_{0}^{l}\left(K\left(w_{1}^{\prime}-\psi_{1}\right)^{2}+E I \psi_{1}^{\prime 2}-g \rho_{1}{w_{1}^{\prime}}^{2} \sin \varphi_{0}\right) d x \\
& =\int_{0}^{l}\left(\frac{K}{2}{w_{1}^{\prime}}^{2}+E I \psi_{1}^{\prime 2}-K \psi_{1}^{2}-g \rho_{1}{w_{1}^{\prime}}^{2} \sin \varphi_{0}\right) d x+\frac{1}{2} \int_{0}^{l} K\left(w_{1}^{\prime}-2 \psi_{1}\right)^{2} d x \\
& \geq \int_{0}^{l}\left(\left(\frac{K}{2}-g \rho_{1} \sin \varphi_{0}\right) w_{1}^{\prime 2}+E I \psi_{1}^{\prime 2}-K \psi_{1}^{2}\right) d x
\end{aligned}
$$

The function $\psi_{1}(x)$ subject to the boundary condition $\psi_{1}(0)=0$ satisfies Friedrichs' inequality of the following form (cf. [13, page 440]):

$$
\int_{0}^{l} \psi_{1}^{2}(x) d x \leq \frac{l^{2}}{2} \int_{0}^{l} \psi_{1}^{\prime 2}(x) d x
$$

Using this inequality in (3.13), we conclude that

$$
\lambda_{1}\left\langle\begin{array}{ll}
w_{1} & w_{1} \\
\psi_{1} & \psi_{1}
\end{array}\right\rangle_{\mathscr{H}} \geq \int_{0}^{l}\left(\left(\frac{K}{2}-g \rho_{1} \sin \varphi_{0}\right) w_{1}^{\prime 2}+\left(E I-\frac{K l^{2}}{2}\right) \psi_{1}^{\prime 2}\right) d x \geq 0,
$$

provided that the conditions (3.9) are satisfied. This proves that all eigenvalues $\lambda$ are nonnegative.

For the rest of this section we assume that $E I, I_{\rho}, K$, and $\rho$ are constants, and that $\sin \varphi_{0}=0$. The coefficients of the Sturm-Liouville problem are constant under this assumption, and, therefore, it is easy to find the general solution of the corresponding system of ODEs. This solution is needed for computing the coefficients of an approximate dynamical model in the sequel (formulae (4.5) define coefficients of the approximate system (4.4) through eigenvalues and eigenfunctions of (3.5)).

We introduce in (3.5) the following dimensionless functions:

$$
\zeta\left(\frac{x}{l}\right)=\frac{\bar{w}(x)}{l}, \quad \theta\left(\frac{x}{l}\right)=\bar{\psi}(x)
$$

and parameters:

$$
p_{1}=\frac{\rho l^{2}}{K}, \quad p_{2}=\frac{K l^{2}}{E I}, \quad p_{3}=\frac{I_{\rho} l^{2}}{E I}, \quad p_{4}=\frac{m l}{K}, \quad p_{5}=\frac{l J_{c}}{E I} .
$$


Then (3.5) is reduced to the following problem:

$$
\begin{gathered}
\frac{d}{d \tau}\left(\begin{array}{c}
\zeta(\tau) \\
\zeta_{\tau}(\tau) \\
\theta(\tau) \\
\theta_{\tau}(\tau)
\end{array}\right)=\left(\begin{array}{cccc}
0 & 1 & 0 & 0 \\
-\lambda p_{1} & 0 & 0 & 1 \\
0 & 0 & 0 & 1 \\
0 & -p_{2} & p_{2}-\lambda p_{3} & 0
\end{array}\right) \times\left(\begin{array}{c}
\zeta(\tau) \\
\zeta_{\tau}(\tau) \\
\theta(\tau) \\
\theta_{\tau}(\tau)
\end{array}\right), \quad \tau=\frac{x}{l} \in(0,1) ; \\
\zeta_{\tau}(1)-\theta(1)=\lambda p_{4} \zeta(1), \quad \theta_{\tau}(1)=\lambda p_{5} \theta(1), \quad \zeta(0)=\theta(0)=0,
\end{gathered}
$$

where $\zeta_{\tau}(\tau)$ and $\theta_{\tau}(\tau)$ stand for derivatives with respect to $\tau$. The eigenvalues and eigenvectors of the matrix in (3.18) are, respectively, given by

$$
\mu_{j}=i \sigma_{j}, \quad v_{j}=\left(\begin{array}{c}
4 \mu_{j}\left(\lambda p_{3}-\sigma_{j}^{2}\right) \\
\lambda c_{3}+4 \lambda p_{1}\left(\sigma_{j}^{2}-\lambda p_{3}\right) \\
\lambda c_{3} \\
\lambda c_{3} \mu_{j}
\end{array}\right), \quad j=1,2,3,4
$$

where

$$
\begin{gathered}
\sigma_{1}=-\sigma_{2}=\frac{\sqrt{2}}{2} \sqrt{c_{1} \lambda-\sqrt{c_{2}{ }^{2} \lambda^{2}+c_{3} \lambda}}, \quad \sigma_{3}=-\sigma_{4}=\frac{\sqrt{2}}{2} \sqrt{c_{1} \lambda+\sqrt{c_{2}{ }^{2} \lambda^{2}+c_{3} \lambda}}, \\
c_{1}=p_{1}+p_{3}, \quad c_{2}=p_{1}-p_{3}, \quad c_{3}=4 p_{1} p_{2} .
\end{gathered}
$$

The general solution of (3.18) therefore reads as

$$
\left(\zeta, \zeta_{\tau}, \theta, \theta_{\tau}\right)^{T}(\tau)=C_{1} v_{1} e^{i \sigma_{1} \tau}+C_{2} v_{2} e^{-i \sigma_{1} \tau}+C_{3} v_{3} e^{i \sigma_{3} \tau}+C_{4} v_{4} e^{-i \sigma_{3} \tau}
$$

By substituting (3.22) into the boundary conditions (3.19), we get a system of linear algebraic equations with respect to (complex) variables $C_{1}, C_{2}, C_{3}, C_{4}$. That system has a nontrivial solution if

$$
\begin{aligned}
& \varkappa(\lambda)= \\
& \left|\begin{array}{cccc}
e^{-i \sigma_{1}} & e^{i \sigma_{1}} & e^{-i \sigma_{3}} & e^{i \sigma_{3}} \\
\sigma_{1}\left(\sigma_{1}^{2}-\lambda p_{3}\right) e^{-i \sigma_{1}} & -\sigma_{1}\left(\sigma_{1}^{2}-\lambda p_{3}\right) e^{i \sigma_{1}} & \sigma_{3}\left(\sigma_{3}^{2}-\lambda p_{3}\right) e^{-i \sigma_{3}} & -\sigma_{3}\left(\sigma_{3}^{2}-\lambda p_{3}\right) e^{i \sigma_{3}} \\
\left(\sigma_{1}^{2}-\lambda p_{3}\right)\left(p_{1}+i p_{4} \sigma_{1}\right) & \left(\sigma_{1}^{2}-\lambda p_{3}\right)\left(p_{1}-i p_{4} \sigma_{1}\right) & \left(\sigma_{3}^{2}-\lambda p_{3}\right)\left(p_{1}+i p_{4} \sigma_{3}\right) & \left(\sigma_{3}^{2}-\lambda p_{3}\right)\left(p_{1}-i p_{4} \sigma_{3}\right) \\
i \sigma_{1}-\lambda p_{5} & i \sigma_{1}+\lambda p_{5} & i \sigma_{3}-\lambda p_{5} & i \sigma_{3}+\lambda p_{5}
\end{array}\right|=0 .
\end{aligned}
$$

The roots of $\varkappa(\lambda)=0$ define the eigenvalues $\lambda$ for the Sturm-Liouville problem (3.5) when its coefficients are constant. It is clear that the function $\varkappa(\lambda)$, given by (3.23), is analytic in its domain of definition. Then the uniqueness theorem for analytic functions implies that either $\varkappa(\lambda) \equiv 0$ or the set of all eigenvalues for (3.5) is discrete. The former is impossible for "typical" values of parameters (see, for example, [13], where the spectrum was estimated for a particular case $p_{1}=p_{2}=p_{3}=1, p_{4}=p_{5}=0$ ). We do not estimate solutions of the characteristic equation (3.23) here. Such a study requires additional assumptions on the mechanical parameters, based on real measurements, and is not of principal interest for this work. 


\section{The Galerkin approximation}

To derive a Galerkin approximation (see, e.g., [6]), we consider a variational formulation of the boundary value problem as follows: if $(\tilde{\varphi}(t), \tilde{w}(x, t), \tilde{\psi}(x, t))(0 \leq x \leq l)$ is a solution of (3.2), corresponding to $M(t)$, on an interval $t \in \mathscr{I} \subset \mathbb{R}$ then

$$
\begin{gathered}
\ddot{\tilde{\varphi}}(t)-v=0, \\
\tilde{\mu}=\int_{0}^{l} \delta \tilde{w}(x, t)\left\{\left(\ddot{\widetilde{w}}+x v-g \tilde{\varphi} \sin \varphi_{0}\right) \rho\right. \\
\left.+\left(K\left(\tilde{\psi}-\tilde{w}^{\prime}\right)+\rho_{1} g\left(\tilde{w}^{\prime} \sin \varphi_{0}+\tilde{\varphi} w_{0}^{\prime} \cos \varphi_{0}\right)\right)^{\prime}+\cdots\right\} d x \\
+\int_{0}^{l} \delta \tilde{\psi}(x, t)\left\{I_{\rho} \ddot{\widetilde{\psi}}+K\left(\tilde{\psi}-\tilde{w}^{\prime}\right)-\left(E I \tilde{\psi}^{\prime}\right)^{\prime}+I_{\rho} v\right\} d x+\left.\delta \tilde{\psi}(l, t)\left\{J_{c} \ddot{\widetilde{\psi}}+E I \tilde{\psi}^{\prime}+J_{c} v\right\}\right|_{x=l} \\
+\left.\delta \tilde{w}(l, t)\left\{K\left(\widetilde{w}^{\prime}-\tilde{\psi}\right)+m\left(\ddot{\widetilde{w}}+l v-g\left(\tilde{\varphi}+\widetilde{w}^{\prime}\right) \sin \varphi_{0}-g \tilde{\varphi} w_{0}^{\prime} \cos \varphi_{0}\right)+\cdots\right\}\right|_{x=l}=0, \quad \forall t \in \mathscr{I},
\end{gathered}
$$

for each admissible variation $(\delta \tilde{w}(x, t), \delta \widetilde{\psi}(x, t))$ satisfying the boundary conditions $\delta \widetilde{w}(0$, $t)=0$ and $\delta \tilde{\psi}(0, t)=0$. (The derivation of $\tilde{\mu}$ from (3.2) uses the standard technique: integration by parts, collecting terms, and so forth. The expression (4.1) may also be obtained by expanding (2.9) in a neighborhood of the equilibrium and neglecting the higher order terms.) Here $v$ is given by the expression (3.3).

Let us fix an integer number $N \geq 1$ and consider nontrivial solutions $\left(\lambda_{j}, w_{j}, \psi_{j}\right)$ of (3.5) for $j=1,2, \ldots, N$. We assume that all $\lambda_{j}$ are different and substitute finite sums

$$
\widetilde{w}(x, t)=\sum_{j=1}^{N} q_{j}(t) w_{j}(x), \widetilde{\psi}(x, t)=\sum_{j=1}^{N} q_{j}(t) \psi_{j}(x)
$$

into (3.3) and (4.1). We also restrict $\delta \widetilde{w}$ and $\delta \tilde{\psi}$ to finite-dimensional subspaces:

$$
\delta \tilde{w}(\cdot, t) \in \operatorname{span}\left\{w_{1}(\cdot), \ldots, w_{N}(\cdot)\right\}, \quad \delta \tilde{\psi}(\cdot, t) \in \operatorname{span}\left\{\psi_{1}(\cdot), \ldots, \psi_{N}(\cdot)\right\}
$$

By assuming $\delta \tilde{w}(x, t)=w_{i}(x)$ and $\delta \tilde{\psi}(x, t)=\psi_{i}(x)$ in $(4.1)$ for $i=1,2, \ldots, N$ and exploiting Lemma 3.1, we obtain the following control system with respect to $\tilde{\varphi}, q_{1}, q_{2}, \ldots, q_{N}$ :

$$
\begin{aligned}
& \dot{z}_{1}=A_{11} z_{1}+A_{12} z_{2}+B_{1} u+R_{1}(z, u), \\
& \dot{z}_{2}=A_{21} z_{1}+A_{22} z_{2}+B_{2} u+R_{2}(z, u), \quad z=\left(z_{1}^{T}, z_{2}^{T}\right)^{T},
\end{aligned}
$$


where $z$ is the state, $u$ is the control,

$$
\begin{aligned}
& z_{1}=(\tilde{\varphi}, \dot{\tilde{\varphi}})^{T}, \quad z_{2}=\left(q_{1}, \dot{q}_{1}, q_{2}, \dot{q}_{2}, \ldots, q_{N}, \dot{q}_{N}\right)^{T}, \\
& u=\frac{\widetilde{M}}{J_{0}+\int_{0}^{l}\left(w_{0}^{2} \rho-w_{0}^{\prime 2} \rho_{2}\right) d x+m w_{0}^{2}(l)}, \\
& A_{11}=\left(\begin{array}{cc}
0 & 1 \\
d_{0} & 0
\end{array}\right), \quad A_{12}=\left(\begin{array}{ccccccc}
0 & 0 & 0 & 0 & \cdots & 0 & 0 \\
d_{1} & 0 & d_{2} & 0 & \cdots & d_{N} & 0
\end{array}\right), \\
& B_{1}=\left(\begin{array}{l}
0 \\
1
\end{array}\right), \quad B_{2}=\left(0,-b_{1}, \ldots, 0,-b_{N}\right)^{T} \\
& A_{21}=\left(\begin{array}{cc}
0 & 0 \\
a_{1}-b_{1} d_{0} & 0 \\
0 & 0 \\
a_{2}-b_{2} d_{0} & 0 \\
\vdots & \vdots \\
0 & 0 \\
a_{N}-b_{N} d_{0} & 0
\end{array}\right), \quad A_{22}=\left(\begin{array}{ccccccc}
0 & 1 & 0 & 0 & \cdots & 0 & 0 \\
-\lambda_{1}-b_{1} d_{1} & 0 & -b_{1} d_{2} & 0 & \cdots & -b_{1} d_{N} & 0 \\
0 & 0 & 0 & 1 & \cdots & 0 & 0 \\
-b_{2} d_{1} & 0 & -\lambda_{2}-b_{2} d_{2} & 0 & \cdots & -b_{2} d_{N} & 0 \\
\vdots & \vdots & \vdots & \vdots & \ddots & \vdots & \vdots \\
0 & 0 & 0 & 0 & \cdots & 0 & 1 \\
-b_{N} d_{1} & 0 & -b_{N} d_{2} & 0 & \cdots & -\lambda_{N}-b_{N} d_{N} & 0
\end{array}\right) \text {, } \\
& a_{j}=g \frac{\left(\int_{0}^{l} \rho w_{j} d x+m w_{j}(l)\right) \sin \varphi_{0}+\int_{0}^{l} \rho_{1} w_{0}^{\prime} w_{j}^{\prime} d x \cos \varphi_{0}}{\int_{0}^{l}\left(\rho w_{j}^{2}+I_{\rho} \psi_{j}^{2}\right) d x+m w_{j}^{2}(l)+J_{c} \psi_{j}^{2}(l)}, \\
& b_{j}=\frac{\int_{0}^{l}\left(\rho x w_{j}+I_{\rho} \psi_{j}\right) d x+m l w_{j}(l)+J_{c} \psi_{j}(l)}{\int_{0}^{l}\left(\rho w_{j}^{2}+I_{\rho} \psi_{j}^{2}\right) d x+m w_{j}^{2}(l)+J_{c} \psi_{j}^{2}(l)} \\
& d_{0}=\frac{\int_{0}^{l}\left[\left(\rho w_{0}-\rho_{1} w_{0}^{\prime}\right) \cos \varphi_{0}-(1 / 2)\left(w_{0}^{\prime}\right)^{2} \rho_{1} \sin \varphi_{0}\right] d x+m w_{0}(l) \cos \varphi_{0}}{J_{0}+\int_{0}^{l}\left(w_{0}^{2} \rho-w_{0}^{\prime 2} \rho_{2}\right) d x+m w_{0}^{2}(l)} g, \\
& d_{j}=\frac{g \int_{0}^{l}\left[\left(w_{0}^{\prime} \cos \varphi_{0}-\sin \varphi_{0}\right) \rho_{1} w_{j}^{\prime}+\rho w_{j} \sin \varphi_{0}\right] d x+m g w_{j}(l) \sin \varphi_{0}+E I \psi_{j}^{\prime}(0)}{J_{0}+\int_{0}^{l}\left(w_{0}^{2} \rho-w_{0}^{\prime 2} \rho_{2}\right) d x+m w_{0}^{2}(l)},
\end{aligned}
$$

the nonlinear term $R(z, u)=\left(R_{1}^{T}, R_{2}^{T}\right)^{T}$ satisfies the estimate

$$
\|R(z, u)\|=O\left(\|z\|^{2}+u^{2}\right)
$$

around the equilibrium point $z=0, u=0$. The control system (4.4) is a finite dimensional approximation of (3.2) corresponding to the flexible coordinates of order up to $N$. 


\section{Stabilization in finite dimensions}

In this section, an explicit procedure for stabilizing controller design is proposed.

Theorem 5.1. Assume that all eigenvalues $\left(\lambda_{1}, \ldots, \lambda_{N}\right)$ are positive and different, and that $a_{j}+\lambda_{j} b_{j} \neq 0$ for each $j=1,2, \ldots, N$. Then system (4.4) is stabilizable by the following feedback control:

$$
\begin{gathered}
u=K z, \quad K=\left(K_{1}, K_{2}\right), \quad K_{1}=\left(-d_{0}-\frac{h_{1}+\sum_{j=1}^{N} a_{j}\left(b_{j}+a_{j} / \lambda_{j}\right)}{h_{2}},-\frac{h_{0}}{h_{2}}\right), \\
K_{2}=\left(-d_{1}+\frac{a_{1}+\lambda_{1} b_{1}}{h_{2}}, 0,-d_{2}+\frac{a_{2}+\lambda_{2} b_{2}}{h_{2}}, 0, \ldots,-d_{N}+\frac{a_{N}+\lambda_{N} b_{N}}{h_{2}}, 0\right),
\end{gathered}
$$

where $h_{0}, h_{1}$, and $h_{2}$ are arbitrary positive constants.

Proof. Consider a Lyapunov function candidate

$$
2 V(z)=\left(h_{1}+\sum_{j=1}^{N} \frac{a_{j}^{2}}{\lambda_{j}}\right) \tilde{\varphi}^{2}+\left(h_{2}+\sum_{j=1}^{N} b_{j}^{2}\right) \dot{\tilde{\varphi}}^{2}+\sum_{j=1}^{N}\left(\lambda_{j} q_{j}^{2}+\dot{q}_{j}^{2}-2 a_{j} \tilde{\varphi} q_{j}+2 b_{j} \dot{\tilde{\varphi}} \dot{q}_{j}\right)
$$

By applying the Cauchy-Schwartz inequality, we get

$$
2 V \geq G_{1}\left(-|\tilde{\varphi}|,\left(\sum_{j=1}^{N} \lambda_{j} q_{j}^{2}\right)^{1 / 2}\right)+G_{2}\left(-|\dot{\tilde{\varphi}}|,\left(\sum_{j=1}^{N} \dot{q}_{j}^{2}\right)^{1 / 2}\right)
$$

where

$$
\begin{aligned}
& G_{1}(\alpha, \beta)=\left(h_{1}+\sum_{j=1}^{N} \frac{a_{j}^{2}}{\lambda_{j}}\right) \alpha^{2}+2\left(\sum_{j=1}^{N} \frac{a_{j}^{2}}{\lambda_{j}}\right)^{1 / 2} \alpha \beta+\beta^{2}, \\
& G_{2}(\alpha, \beta)=\left(h_{2}+\sum_{j=1}^{N} b_{j}^{2}\right) \alpha^{2}+2\left(\sum_{j=1}^{N} b_{j}{ }^{2}\right)^{1 / 2} \alpha \beta+\beta^{2} .
\end{aligned}
$$

Sylvester's criterion for quadratic forms $G_{1}$ and $G_{2}$ implies that both $G_{1}$ and $G_{2}$ are positive definite if $h_{1}>0$ and $h_{2}>0$. Then the quadratic form $V$ is positive definite due to estimate (5.3).

The time-derivative of $V$ along the trajectories of the linear part of (4.4) is

$$
\dot{V}=h_{2} \dot{\tilde{\varphi}} v+\left(h_{1}+h_{2} d_{0}+\sum_{j=1}^{N} a_{j}\left(\frac{b_{j}+a_{j}}{\lambda_{j}}\right)\right) \tilde{\varphi} \dot{\tilde{\varphi}}+\dot{\tilde{\varphi}} \sum_{j=1}^{N} q_{j}\left(h_{2} d_{j}-a_{j}-\lambda_{j} b_{j}\right) \text {. }
$$

We choose a constant $h_{0}>0$ arbitrarily and define the feedback control in order to have $\dot{V}=-h_{0} \dot{\tilde{\varphi}}^{2}$. This yields expression (5.1). 
Now we apply the Barbashin-Krasovskii theorem (or LaSalle's invariance principle, cf. [21]). For this purpose consider the set

$$
Z_{0}=\left\{\left(\tilde{\varphi}, \dot{\widetilde{\varphi}}, q_{1}, \dot{q}_{1}, \ldots, q_{N}, \dot{q}_{N}\right) \in \mathbb{R}^{2 N+2}: \dot{V}=0\right\}
$$

Each positive semitrajectory of the linear approximation of (4.4) with (5.1) on $Z_{0}$ satisfies the following relations:

$$
\begin{gathered}
\ddot{q}_{j}=-\lambda_{j} q_{j}+a_{j} \tilde{\varphi}, \\
\sum_{j=1}^{N}\left[-a_{j} b_{j} \tilde{\varphi}+\left(a_{j}+\lambda_{j} b_{j}\right) q_{j}\right]=\left(h_{1}+\sum_{j=1}^{N} \frac{a_{j}^{2}}{\lambda_{j}}\right) \tilde{\varphi}=\text { const }, \quad t \geq 0 .
\end{gathered}
$$

The above relations imply

$$
\sum_{j=1}^{N}\left(a_{j}+\lambda_{j} b_{j}\right)\left(r_{1 j} \cos \left(\sqrt{\lambda_{j}} t\right)+r_{2 j} \sin \left(\sqrt{\lambda_{j}} t\right)\right)=h_{1} \tilde{\varphi}
$$

for some constants $r_{1 j}, r_{2 j}$, and $\tilde{\varphi}$. Exploiting the fact that

$$
\left\{1, \sin \left(\sqrt{\lambda_{j}} t\right), \cos \left(\sqrt{\lambda_{j}} t\right)\right\}_{j=1}^{N}
$$

are linearly-independent functions on $[0,+\infty)$ (cf. [22]), we get that (5.8) is possible only if $\tilde{\varphi}=0$ and $r_{1 j}=r_{2 j}=0$ for all $j=1,2, \ldots, N$. Thus, the only semitrajectory of the linearized closed-loop system on $Z_{0}$ is the trivial one, and the trivial solution of the linear part of (4.4), (5.1) is asymptotically stable by the Barbashin-Krasovskii theorem (LaSalle's invariance principle). Now local asymptotic stability of the nonlinear closed-loop system follows from Lyapunov's theorem on stability using linearization.

Remark 5.2. As it follows from the representation $\dot{V}=-h_{0} \dot{\tilde{\varphi}}^{2}$, the choice of constant $h_{0}$ affects the decay rate of the Lyapunov function along trajectories of the closed-loop system. On the one hand, the more $h_{0}$ the faster convergence of solutions to the equilibrium could be achieved (for solutions with $\dot{\widetilde{\varphi}} \neq 0$ ). On the other hand, for large $h_{0}$, the gain $-h_{0} / h_{2}$, appearing in formula (5.1), may take large values if $h_{2}$ is small. This suggests us to choose $h_{0}$ as maximal as possible, and to select $h_{2}$ in such a way that the term $-\left(h_{0} / h_{2}\right) \dot{\tilde{\varphi}}$, appearing in $u=K z$, would not bring the control input $u$ to its saturation bound (for typical disturbances $\dot{\tilde{\varphi}}$ ). The constant $h_{1}$ should be then defined according to a desired geometry of the level surfaces for the quadratic form $V$. Indeed, constants $h_{1}$ and $h_{2}$ define a relation between semiaxes for the ellipsoids $V(z)=$ const, and hence a desired ratio between overshoots for $\tilde{\varphi}$ and $\dot{\tilde{\varphi}}$ can be estimated in terms of $h_{1}$ and $h_{2}$. Certainly, this suggestion is based on the linearized system and does not give a rigorous characterization of the global behavior.

\section{Observer design}

In order to implement the feedback law (5.1) in practice, one should reconstruct the complete state vector of (4.4) from the outputs which can be measured. The values of $w(x, t)$ 
and $\psi(x, t)$ cannot be directly estimated in a real flexible manipulator. Instead, there is a set of strain gauges located at a point $x=l_{0}, 0 \leq l_{0} \leq l$, which allows measurement of some components of the strain tensor. By using only the principal part of the strain at $x=l_{0}$, we get the output $\left.\psi^{\prime}(x, t)\right|_{x=l_{0}}$ for each $t \geq 0$. By subtracting from the signals $\varphi(t)$ and $\left.\psi^{\prime}(x, t)\right|_{x=l_{0}}$ their steady-state values and rescaling, we assume that the following outputs are available for the finite dimensional approximation (4.4):

$$
y_{1}(t)=\tilde{\varphi}(t), \quad y_{2}(t)=\left.l^{2} \widetilde{\psi}^{\prime}(x, t)\right|_{x=l_{0}}=\sum_{j=1}^{N} \chi_{j} q_{j}(t),
$$

where $\chi_{j}=l^{2} \psi_{j}^{\prime}\left(l_{0}\right)$. We introduce the factor $l^{2}$ in order to get the dimension of length for the output $y_{2}$.

Let us rewrite the output (6.1) as follows:

$$
y_{1}=C_{1} z_{1}, \quad y_{2}=C_{2} z_{2}, \quad C_{1}=(1,0), \quad C_{2}=\left(\chi_{1}, 0, \chi_{2}, 0, \ldots, \chi_{N}, 0\right)
$$

Lemma 6.1. The control system (4.4), (6.2) is locally observable at $z=0$ if

$$
\left|\begin{array}{cccc}
\pi_{11} & \pi_{12} & \cdots & \pi_{1 N} \\
\pi_{21} & \pi_{22} & \cdots & \pi_{2 N} \\
\vdots & \vdots & \ddots & \vdots \\
\pi_{N 1} & \pi_{N 2} & \cdots & \pi_{N N}
\end{array}\right| \neq 0
$$

where $\pi_{1, j}=\chi_{j}, \pi_{k, j}=-\lambda_{j} \pi_{k-1, j}-d_{j} \sum_{i=1}^{N} \pi_{k-1, i} b_{i}, j=\overline{1, N}, k=\overline{2, N}$.

In particular, the condition (6.3) is equivalent to $\chi_{1} \neq 1$ if $N=1$ or

$$
\chi_{1} \chi_{2}\left(\lambda_{1}-\lambda_{2}+b_{1} d_{1}-b_{2} d_{2}\right)+b_{2} \chi_{2}^{2} d_{1}-b_{1} \chi_{1}^{2} d_{2} \neq 0 \quad \text { if } N=2 .
$$

Proof. The linear part of (4.4), (6.2) can be written in terms of output $y_{1}$ as follows:

$$
\begin{aligned}
& z_{1}=\left(y_{1}, \dot{y}_{1}\right)^{T}, \\
& \dot{z}_{2}=A_{22} z_{2}+B_{2} u+\left(0, a_{1}-b_{1} d_{0}, 0, a_{2}-b_{2} d_{0}, \ldots, 0, a_{N}-b_{N} d_{0}\right)^{T} y_{1}, \\
& y_{2}=C_{2} z_{2} .
\end{aligned}
$$

Hence, the above system is observable if the pair $\left(A_{22}, C_{2}\right)$ satisfies the Kalman observability condition (cf. [23, Theorem 3.1, page 58]):

$$
\operatorname{rank}\left(\begin{array}{c}
C_{2} \\
C_{2} A_{22} \\
\vdots \\
C_{2} A_{22}^{2 N-1}
\end{array}\right)=2 N
$$


Straightforward computations show that

$$
\operatorname{det}\left(\begin{array}{c}
C_{2} \\
C_{2} A_{22} \\
\vdots \\
C_{2} A_{22}^{2 N-1}
\end{array}\right)=\left|\begin{array}{cccc}
\pi_{11} & \pi_{12} & \cdots & \pi_{1 N} \\
\pi_{21} & \pi_{22} & \cdots & \pi_{2 N} \\
\vdots & \vdots & \ddots & \vdots \\
\pi_{N 1} & \pi_{N 2} & \cdots & \pi_{N N}
\end{array}\right|^{2}
$$

Therefore, (6.3) implies the observability rank condition for the linear part of (4.4), (6.2). It also means that (4.4), (6.2) is strongly locally observable at $z=0$ by the HermannKrener theorem [24].

The following theorem gives an explicit procedure for the Luenberger-type observer design.

Theorem 6.2. Suppose that the control system (4.4), (6.2) satisfies the observability condition (6.3), all $\lambda_{j}$ are positive and different, $a_{j}+\lambda_{j} b_{j} \neq 0$, and $b_{j} d_{j}>0$ for $j=\overline{1, N}$. Then the origin $z=0, \bar{z}=0$ of the extended system (4.4), (6.2) and

$$
\begin{gathered}
\dot{\bar{z}}_{1}=\left(A_{11}-F_{1} C_{1}\right) \bar{z}_{1}+A_{12} \bar{z}_{2}+F_{1} y_{1}+B_{1} u \\
\dot{\bar{z}}_{2}=\left(A_{22}-F_{22} C_{2}\right) \bar{z}_{2}+F_{21} y_{1}+F_{22} y_{2}+B_{2} u
\end{gathered}
$$

with $u=K \bar{z}$ is locally asymptotically stable, where $K$ is given by (5.1),

$$
\begin{gathered}
F_{1}=\left(\phi_{1}, d_{0}+\phi_{2}\right)^{T}, \\
F_{22}=\left(f_{1}, 0, f_{2}, 0, \ldots, f_{N}, 0\right)^{T}, \\
Q=\left(\begin{array}{cccc}
\frac{\lambda_{1} d_{1}}{b_{1}}+d_{1}^{2} & d_{1} d_{2} & \ldots & d_{1} d_{N} \\
d_{2} d_{1} & \frac{\lambda_{2} d_{2}}{b_{2}}+d_{2}^{2} & \ldots & d_{2} d_{N} \\
\vdots & \vdots & \ddots & \vdots \\
d_{N} d_{1} & d_{N} d_{2} & \ldots & \frac{\lambda_{N} d_{N}}{b_{N}}+d_{N}^{2}
\end{array}\right)
\end{gathered}
$$

Here $\phi_{1}, \phi_{2}$, and $\gamma$ are any positive constants.

Proof. Consider the observation errors $e_{1}=z_{1}-\bar{z}_{1}, e_{2}=z_{2}-\bar{z}_{2}$. Then subtracting (6.8) from (4.4) yields the following dynamics:

$$
\dot{e}_{1}=H_{1} e_{1}+A_{12} e_{2}+R_{1}(z, u), \quad \dot{e}_{2}=H_{2} e_{2}+R_{2}(z, u)
$$

here $H_{1}=A_{11}-F_{1} C_{1}$ and $H_{2}=A_{22}-F_{22} C_{2}$. We see that the roots of the polynomial

$$
\operatorname{det}\left(H_{1}-\mu I\right)=\left|\begin{array}{cc}
-\phi_{1}-\mu & 1 \\
-\phi_{2} & -\mu
\end{array}\right|=\mu^{2}+\phi_{1} \mu+\phi_{2}
$$


have negative real parts if and only if $\phi_{1}>0$ and $\phi_{2}>0$. Our goal is to show that the real parts of all eigenvalues of

$$
H_{2}=\left(\begin{array}{ccccccc}
-f_{1} \chi_{1} & 1 & -f_{1} \chi_{2} & 0 & \cdots & -f_{1} \chi_{N} & 0 \\
-\lambda_{1}-b_{1} d_{1} & 0 & -b_{1} d_{2} & 0 & \cdots & -b_{1} d_{N} & 0 \\
-f_{2} \chi_{1} & 0 & -f_{2} \chi_{2} & 1 & \cdots & -f_{2} \chi_{N} & 0 \\
-b_{2} d_{1} & 0 & -\lambda_{2}-b_{2} d_{2} & 0 & \cdots & -b_{2} d_{N} & 0 \\
\vdots & \vdots & \vdots & \vdots & \ddots & \vdots & \vdots \\
-f_{N} \chi_{1} & 0 & -f_{N} \chi_{2} & 0 & \cdots & -f_{N} \chi_{N} & 1 \\
-b_{N} d_{1} & 0 & -b_{N} d_{2} & 0 & \cdots & -\lambda_{N}-b_{N} d_{N} & 0
\end{array}\right)
$$

are also negative if the conditions of Theorem 6.2 are satisfied. Let us denote $e_{2}=\left(\xi_{1}, \eta_{1}\right.$, $\left.\ldots, \xi_{N}, \eta_{N}\right)^{T}$ and consider the following quadratic form:

$$
2 W\left(e_{2}\right)=\sum_{j=1}^{N} \frac{d_{j} \eta_{j}^{2}}{b_{j}}+\left(\xi_{1}, \xi_{2}, \ldots, \xi_{N}\right) Q\left(\xi_{1}, \xi_{2}, \ldots, \xi_{N}\right)^{T} .
$$

This form is positive definite as $\lambda_{j}>0$ and $b_{j} d_{j}>0$. Indeed, all principal minors $\Delta_{j}$ of $Q$ are positive:

$$
\Delta_{j}=\frac{\left(\lambda_{1} d_{1}\right)\left(\lambda_{2} d_{2}\right) \cdots\left(\lambda_{j} d_{j}\right)}{b_{1} b_{2} \cdots b_{j}}\left(1+\sum_{i=1}^{j} \frac{b_{i} d_{i}}{\lambda_{i}}\right)>0, \quad j=\overline{1, N}
$$

Then Sylvester's criterion implies that $W$ is positive definite. The inequality $\operatorname{det}(Q)=$ $\Delta_{N}>0$ also proves invertibility of $Q$ in (6.9). By computing the time derivative of $W$ along the trajectories of the linear system $\dot{e}_{2}=\mathrm{H}_{2} e_{2}$, we get

$$
\dot{W}\left(e_{2}\right)=-\gamma\left(C_{2} e_{2}\right)^{2} \leq 0
$$

provided that $F_{22}$ is defined by (6.9). As the time derivative of $W$ is negative semi-definite and vanishes on $\operatorname{ker} C_{2}=\left\{e_{2} \in \mathbb{R}^{2 N}: C_{2} e_{2}=0\right\}$, we check whether the linear system $\dot{e}_{2}=$ $\mathrm{H}_{2} e_{2}$ admits a nontrivial semitrajectory on $\operatorname{ker} C_{2}$. Let $C_{2} e_{2}(t) \equiv 0, t \geq 0$, then

$$
\frac{d^{k}}{d t^{k}} C_{2} e_{2}(t)=C_{2}\left(A_{22}-F_{22} C_{2}\right)^{k} e_{2}(t)=C_{2} A_{22}^{k} e_{2}(t)=0, \quad t \geq 0, k \geq 0 .
$$

This implies that, for each $t \geq 0, e_{2}(t)$ is a solution of the following system of linear algebraic equations:

$$
C_{2} A_{22}^{k} e_{2}(t)=0, \quad k=\overline{0,2 N-1} .
$$

The above system has only the trivial solution $e_{2}(t)=0$ because of the observability rank condition (6.3). This proves asymptotic stability of the linear system $\dot{e}_{2}=H_{2} e_{2}$ by the Barbashin-Krasovskii theorem (LaSalle's invariance principle). 
We have shown that the matrices $H_{1}$ and $H_{2}$ are Hurwitz. The nonlinear closed-loop system (4.4), (6.2), (6.8) with $u=K \bar{z}$ can be written in variables $(z, e)$ as follows:

$$
\left(\begin{array}{c}
\dot{z} \\
\dot{e}_{1} \\
\dot{e}_{2}
\end{array}\right)=\left(\begin{array}{ccc}
H_{0} & -B K_{1} & -B K_{2} \\
0 & H_{1} & A_{12} \\
0 & 0 & H_{2}
\end{array}\right)\left(\begin{array}{c}
z \\
e_{1} \\
e_{2}
\end{array}\right)+\left(\begin{array}{c}
R(z, K(z-e)) \\
R_{1}(z, K(z-e)) \\
R_{2}(z, K(z-e))
\end{array}\right),
$$

where

$$
H_{0}=\left(\begin{array}{ll}
A_{11}+B_{1} K_{1} & A_{12}+B_{1} K_{2} \\
A_{21}+B_{2} K_{1} & A_{22}+B_{2} K_{2}
\end{array}\right), \quad B=\left(B_{1}^{T}, B_{2}^{T}\right)^{T} .
$$

As $a_{j}+\lambda_{j} b_{j} \neq 0$ then the conditions of Theorem 5.1 are satisfied and $H_{0}$ is Hurwitz. Hence, the trivial solution of (6.18) is asymptotically stable by linear approximation as the spectrum of its matrix is the union of spectra of the Hurwitz matrices $H_{0}, H_{1}$, and $\mathrm{H}_{2}$.

\section{Conclusions}

We have proposed a feedback controller that stabilizes the equilibrium of a Galerkin approximation for a rotating Timoshenko beam, provided that measurements of the raising angle and the strain at a point are available. The feedback law and coefficients of the dynamical observer are computed explicitly for any number of modal coordinates. A potential field of application of these results is the control design for fire-rescue turntable ladders. An advantage of our approach is that the identification procedure can be reduced significantly in comparison with a multibody model. In addition, the higher modes can be calculated explicitly, which is important for the design of an oscillation damping control of a turntable ladder. For a possible implementation of the controller, it is necessary to integrate a system of ordinary differential equations in real time. We do not consider here such issues as spillover analysis, convergence of Galerkin approximations, computational complexity, or limitation of the sampling rate with respect to the calculation time leaving these problems for future work.

\section{Acknowledgments}

This work was supported in part by the Alexander von Humboldt Foundation and under Grant of the President of Ukraine for Young Scientists. The authors express gratitude to anonymous reviewers for their valuable comments.

\section{References}

[1] Z.-H. Luo, B.-Z. Guo, and O. Morgul, Stability and Stabilization of Infinite Dimensional Systems with Applications, Communications and Control Engineering Series, Springer, London, UK, 1999.

[2] H. A. Talebi, R. V. Patel, and K. Khorasani, Control of Flexible-Link Manipulators Using Neural Networks, Springer, London, UK, 2001. 
[3] O. Sawodny, H. Aschemann, and A. Bulach, "Mechatronical designed control of fire-rescue turntable ladders as flexible link robots," in Proceedings of the 15th IFAC World Congress, Barcelona, Spain, July 2002, CD-ROM file 385.pdf.

[4] K. Grosh and P. M. Pinsky, "Design of Galerkin generalized least squares methods for Timoshenko beams," Computer Methods in Applied Mechanics and Engineering, vol. 132, no. 1-2, pp. 1-16, 1996.

[5] A. Zuyev and O. Sawodny, "Control design for Galerkin approximations of a flexible structure," in Proceedings of IFAC Workshop on Generalized Solutions in Control Problems (GSCP '04), pp. 311-318, Fizmatlit, Pereslavl-Zalessky, Russia, September 2004.

[6] J. Donea and A. Huerta, Finite Element Methods for Flow Problems, John Wiley \& Sons, Chichester, UK, 2003.

[7] J. E. Lagnese and G. Leugering, "Controllability of thin elastic beams and plates," in The Control Handbook, W. S. Levine, Ed., pp. 1139-1156, CRC Press-IEEE Press, Boca Raton, Fla, USA, 1996.

[8] I. Lasiecka and R. Triggiani, Control Theory for Partial Differential Equations: Continuous and Approximation Theories. II: Abstract Hyperbolic-Like Systems Over a Finite Time Horizon, vol. 75 of Encyclopedia of Mathematics and Its Applications, Cambridge University Press, Cambridge, UK, 2000.

[9] C.-Z. Xu and J. Baillieul, "Stabilizability and stabilization of a rotating body-beam system with torque control," IEEE Transactions on Automatic Control, vol. 38, no. 12, pp. 1754-1765, 1993.

[10] A. Zuyev, "Partial asymptotic stabilization of nonlinear distributed parameter systems," Automatica, vol. 41, no. 1, pp. 1-10, 2005.

[11] S. P. Timoshenko, "On the correction for shear of the differential equation for transverse vibrations of prismatic bars," Philisophical Magazine, vol. 41, pp. 744-746, 1921, reprinted in: The Collected Papers of S. P. Timoshenko, McGraw-Hill, London, UK, 1953.

[12] J. U. Kim and Y. Renardy, "Boundary control of the Timoshenko beam," SIAM Journal on Control and Optimization, vol. 25, no. 6, pp. 1417-1429, 1987.

[13] W. Krabs and G. M. Sklyar, "On the controllability of a slowly rotating Timoshenko beam," Journal for Analysis and Its Applications, vol. 18, no. 2, pp. 437-448, 1999.

[14] W. Krabs and G. M. Sklyar, "On the stabilizability of a slowly rotating Timoshenko beam," Zeitschrift für Analysis und ihre Anwendungen, vol. 19, no. 1, pp. 131-145, 2000.

[15] Ö. Morgül, "Boundary control of a Timoshenko beam attached to a rigid body: planar motion," International Journal of Control, vol. 54, no. 4, pp. 763-791, 1991.

[16] S. W. Taylor, "A smoothing property of a hyperbolic system and boundary controllability," Journal of Computational and Applied Mathematics, vol. 114, no. 1, pp. 23-40, 2000.

[17] S. W. Taylor and S. C. B. Yau, "Boundary control of a rotating Timoshenko beam," Australian \& New Zealand Industrial and Applied Mathematics Journal, vol. 44, part E, pp. 143-184, 2003.

[18] D.-H. Shi, S. H. Hou, and D.-X. Feng, "Feedback stabilization of a Timoshenko beam with an end mass," International Journal of Control, vol. 69, no. 2, pp. 285-300, 1998.

[19] M. Tadi, "Computational algorithm for controlling a Timoshenko beam," Computer Methods in Applied Mechanics and Engineering, vol. 153, no. 3-4, pp. 153-165, 1998.

[20] A. Zuyev and O. Sawodny, "Observer design for a flexible manipulator model with a payload," in Proceedings of the 45th IEEE Conference on Decision and Control, pp. 4490-4495, San Diego, Calif, USA, December 2006.

[21] N. Rouche, P. Habets, and M. Laloy, Stability Theory by Liapunov's Direct Method, vol. 22 of Applied Mathematical Sciences, Springer, New York, NY, USA, 1977.

[22] W. Krabs, On Moment Theory and Controllability of One-Dimensional Vibrating Systems and Heating Processes, vol. 173 of Lecture Notes in Control and Information Sciences, Springer, Berlin, Germany, 1992. 
[23] W. M. Wonham, Linear Multivariable Control: A Geometric Approach, vol. 10 of Applications of Mathematics (New York), Springer, New York, NY, USA, 3rd edition, 1985.

[24] R. Hermann and A. J. Krener, "Nonlinear controllability and observability," IEEE Transactions on Automatic Control, vol. 22, no. 5, pp. 728-740, 1977.

Alexander Zuyev: Institute of Applied Mathematics \& Mechanics, National Academy of Sciences of Ukraine, R. Luxembourg 74, 83114 Donetsk, Ukraine

Email address: al_zv@mail.ru

Oliver Sawodny: Institute for System Dynamics, Universität Stuttgart, Pfaffenwaldring 9, 70569 Stuttgart, Germany

Email address: sawodny@isys.uni-stuttgart.de 


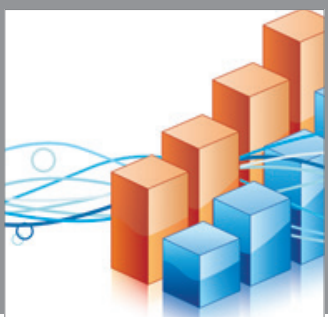

Advances in

Operations Research

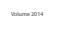

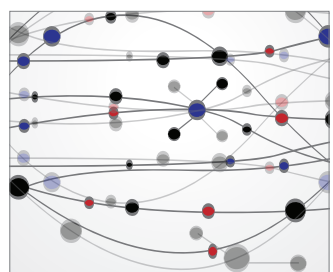

\section{The Scientific} World Journal
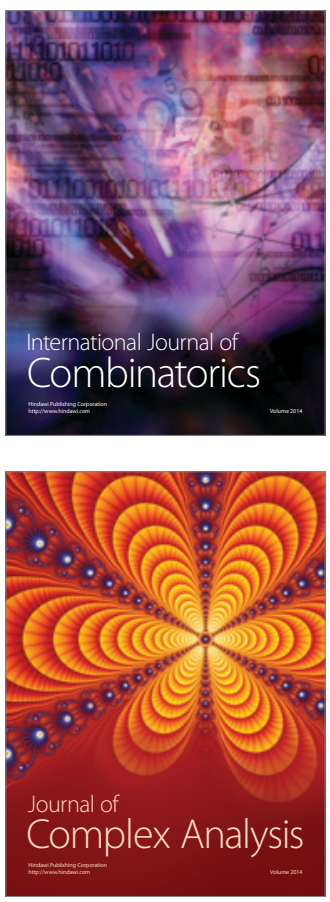

International Journal of

Mathematics and

Mathematical

Sciences
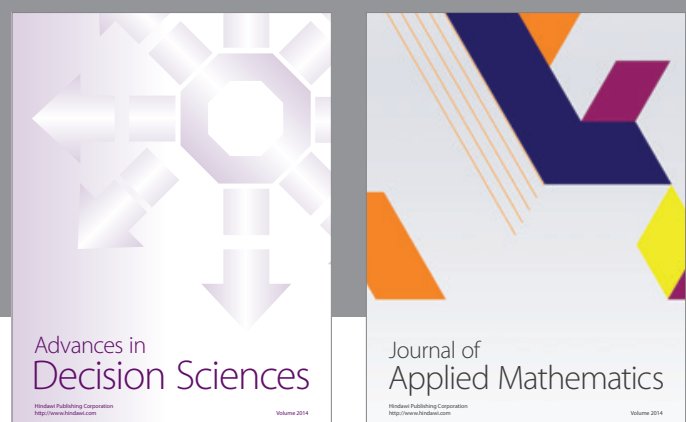

Journal of

Applied Mathematics
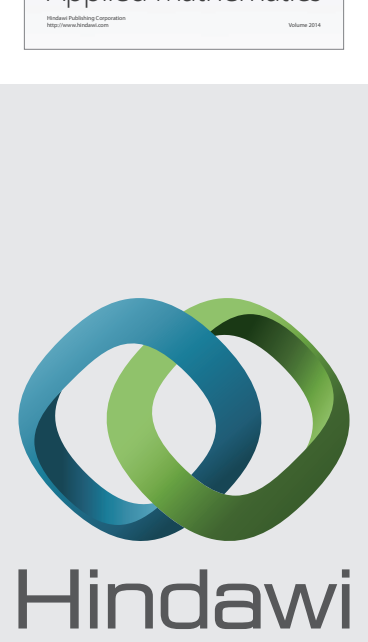

Submit your manuscripts at http://www.hindawi.com
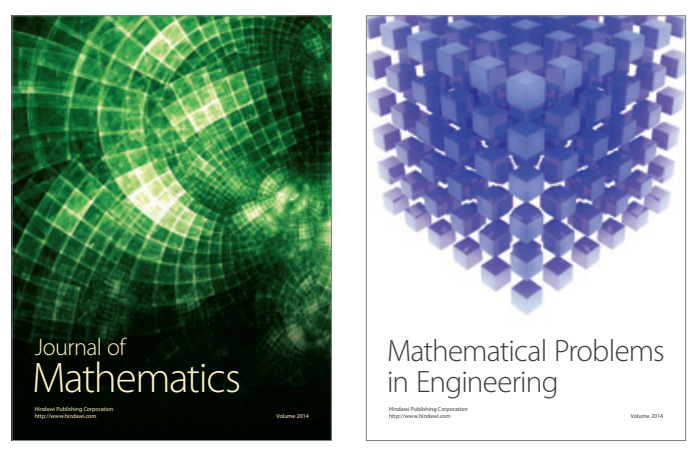

Mathematical Problems in Engineering
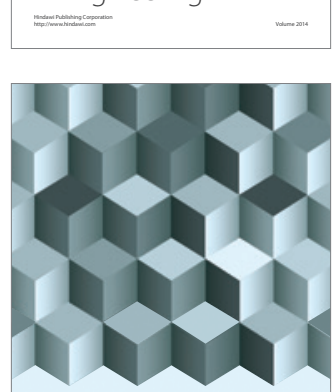

Journal of

Function Spaces
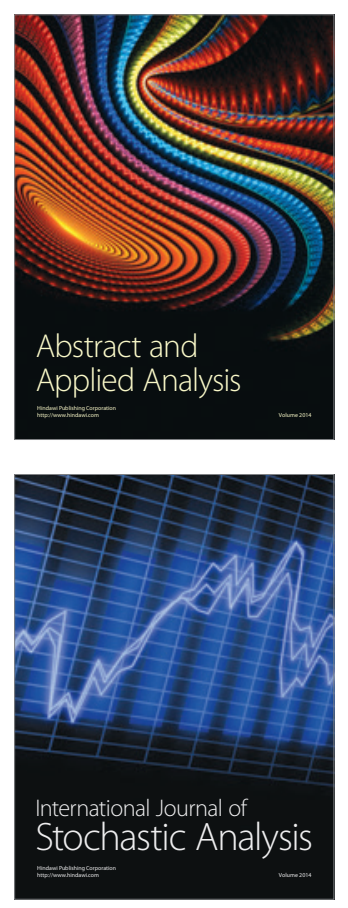

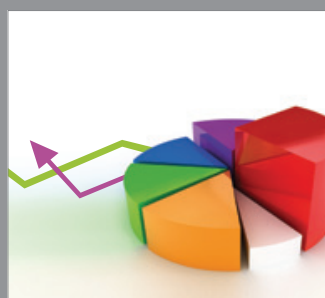

ournal of

Probability and Statistics

Promensencen
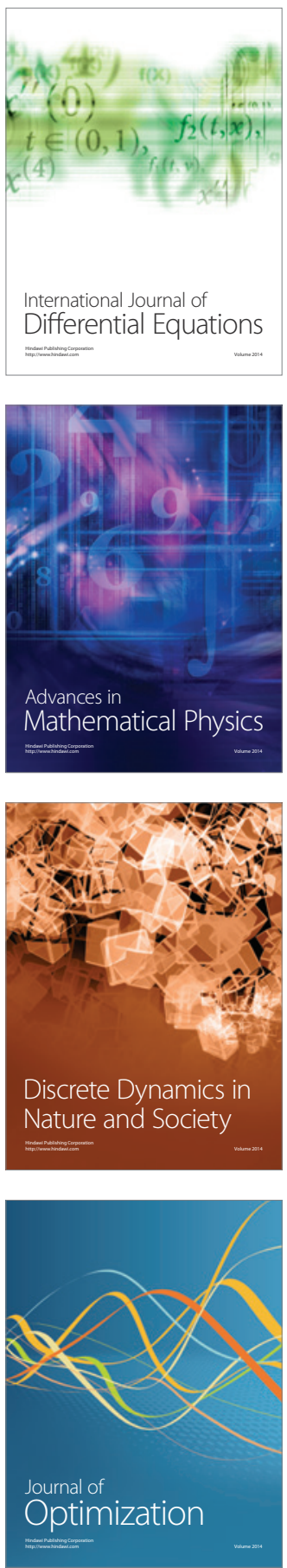